\title{
Role of Nuclearity-Dependent Properties of Small Clusters in the Nucleation Process and Thermal Hysteresis at the Phase Transition ${ }^{\dagger}$
}

\author{
J. Belloni* and P. Pernot \\ Laboratoire de Chimie Physique, UMR CNRS-UPS 8000, Bât. 349, Université Paris-Sud, 91405 Orsay, France
}

Received: February 14, 2003; In Final Form: May 19, 2003

\begin{abstract}
A phenomenological approach is proposed that takes into account the nuclearity-dependent binding energy in a cluster to determine its influence on the earliest steps of the homogeneous nucleation process and to determine the limits of the metastability/supersaturation domain for the monomer-condensed phase transition. It appears that the temperature at which monomers and clusters $\mathbf{M}_{n}$ have equal Gibbs potentials increases with nuclearity $n$ and that the transition temperatures from the monomer to the dimer and to the bulk, respectively, bracket this metastability domain.
\end{abstract}

\section{Introduction}

The phenomenon of homogeneous nucleation, that is, the birth of quite small nuclei of a new phase in the bulk of the mother phase during a first-order transition, has been recognized for a long time to govern the transition rate and the final structural properties of the new phase. This discontinuous change in the condensation of a low-density gas or in the crystallization from dilute solutions is driven by lowering the system free energy. However, close to the equilibrium transition point, the initial phase may remain metastable and large deviations from equilibrium may be observed before the second and more thermodynamically stable phase is formed. This hysteresis, supercooling or supersaturation, is of great importance in materials science and metallurgy because crystallization and condensation are often used as purification and material elaboration steps, or in atmospheric sciences because they govern the weather, the formation of clouds and mist, of the snow and rain falls, of glazed frost, etc.

Since the pioneering works on homogeneous nucleation by Smoluchowski, ${ }^{1}$ Volmer and Weber, ${ }^{2}$ Becker and Doring, ${ }^{3}$ Zeldovich, ${ }^{4}$ and Turnbull and Fisher, ${ }^{5}$ an intense research activity has been steadily developed in this important and difficult field. ${ }^{6-9}$ The classical theory describes the mechanism by which, in the absence of any external germ, an initial critical nucleus should be formed from local energy fluctuations and should grow by gaining successively monomers from the mother phase. For moderate supersaturation conditions, the critical nucleus is found to contain several tens of atoms or molecules. It is therefore considered as a macroscopic entity and the surface energy concept at the phase interface is used. But the kinetics of the nucleus formation from monomers and smaller oligomers is not explicitly considered. The classical theory is in fact more appropriate for heterogeneous nucleation in systems containing preexisting nuclei. At very high supersaturations of homogeneous phases, it is clear that the nucleus size is of a few atoms only and that the specific properties of these clusters have also to be taken into account. An atomistic approach of nucleation has been already applied ${ }^{10}$ to the sudden condensation in

\footnotetext{
† Part of the special issue "Arnim Henglein Festschrift".

* To whom correspondence should be addressed. E-mail: jacqueline.belloni@1cp.u-psud.fr.
}

molecular beam experiments in which the temperature is so cold that the reverse flow of evaporation of the molecules is many orders of magnitude less than the condensation flow and was neglected. Recently, molecular dynamics models have also been developed that consider a limited number of molecules starting with a uniform supersaturated configuration and then submitted to variable conditions of temperature/pressure. ${ }^{11-14}$

Since the 1960s, experimental time-resolved studies in nanosecond range have been performed using the pulse radiolysis method for the coalescence kinetics of metal atoms into clusters in solution under room temperature conditions, the atoms being suddenly produced by a fast radiolytic reduction of ions just after the pulse and constituting indeed a supersaturated phase. The kinetics of the silver clusters formation has been, for example, extensively studied in water and nonaqueous solvents. ${ }^{15-18}$ Similar studies have been performed about the coalescence of $\mathrm{CdS},{ }^{19,20} \mathrm{AgI},{ }^{21}$ or $\mathrm{AgBr}^{22}$ monomers. At room temperature, these systems are far below a possible metastable supersaturated domain with respect to the metal/semiconductor condensation, and no hysteresis was ever observed. On the other hand, the radiation chemistry methods permitted establishment of the nuclearity dependence of some properties of the smallest metal oligomers in solution. ${ }^{23-26}$ This dependence, which was previously predicted by Kubo, ${ }^{27}$ was also confirmed experimentally and theoretically in the gas phase. ${ }^{28-32}$ It was also demonstrated by pulse radiolysis that the critical nuclearity required for the photographic development of silver clusters in solution was not a consequence of supersaturated conditions of a phase-transition model, as previously proposed. ${ }^{33,34}$ In fact, the critical value has been found to result from thermodynamics, specially from the nuclearity dependence of the cluster redox potential and from the redox potential threshold imposed by the developer. ${ }^{35,36}$

The aim of the present work is to consider phenomenologically whether the rate-determining step in the first-order phase transition is similarly dominated by the thermodynamics of the smallest oligomers, how their nuclearity-dependent properties govern the start and rate of nucleation of a population of monomers initially present in a phase under supersaturated conditions, and which are the parameters governing the extent of the metastability domain. 


\section{Phenomenological Cluster Nucleation Model}

According to the classical theory, we consider how a lowdensity population of monomers $\mathbf{M}_{1}$ (in a vapor or a dilute solution) starts after diffusion to agglomerate (by condensation or precipitation) into growing clusters. The pressure is supposed constant, and the process is governed by the temperature. Because of the negligible probability of multiple encounters, the cluster build-up starts necessarily, if the thermodynamical conditions are suitable, by the formation of dimers, followed by the dimer growth. The concentration of monomers is during these initial steps much higher than that of any other oligomers. At this stage, the aggregation reactions can be thus limited to the successive adjunctions of monomers to the growing oligomers.

$$
\begin{gathered}
\mathrm{M}_{1}+\mathrm{M}_{1} \rightleftarrows \mathrm{M}_{2} \\
\mathrm{M}_{2}+\mathrm{M}_{1} \rightleftarrows \mathrm{M}_{3} \\
\mathrm{M}_{3}+\mathrm{M}_{1} \rightleftarrows \mathrm{M}_{4} \\
\mathrm{M}_{n}+\mathrm{M}_{1} \rightleftarrows \mathrm{M}_{n+1} \\
\mathrm{M}_{\infty-1}+\mathrm{M}_{1} \rightleftarrows \mathrm{M}_{\infty}
\end{gathered}
$$

Simultaneously, at each step, back reactions of evaporation or redissolution occur, which correspond to the loss of one monomer. If diffusion-controlled, the forward rate constant of condensation, $k_{\text {cond }}$, is likely independent of $n .{ }^{1}$ In contrast, the evaporation rate constant, $k_{\text {evap }}$, is strongly dependent on the binding energy of a monomer $\mathbf{M}_{1}$ detaching from $\mathbf{M}_{n}$, that is, strongly dependent on $n$.

The phase transition of condensation consists of the replacement of the diluted phase of monomers by the condensed phase via the mechanism $1-5$ in which the dimers and higher oligomers are short-lived transient states. We do not consider in this work whether the clusters possess solid- or liquid-like structures because the transition of the smallest oligomers between different structural arrangements (isomers) differing in energy was predicted to extend over a broad temperature domain. ${ }^{14}$ Far below or far above the bulk transition temperature, the dilute phase (monomers) or the condensed phase, respectively, is stable. At the transition temperature, the monomers and the condensed phase are in equilibrium. In fact, at this temperature, which is the transition point of the bulk, $T_{1 \leftrightarrow \infty}$, the forward reaction rate of condensation of monomers and the backward reaction rate of evaporation (dissolution) are equal. The overall free energy change, $\Delta G_{1 \leftrightarrow \infty}$, between the two coexisting phases is zero at $T_{1 \leftrightarrow \infty}$ :

$$
\Delta G_{1 \leftrightarrow \infty}=\Delta H_{1 \leftrightarrow \infty}-T_{1 \leftrightarrow \infty} \Delta S_{1 \leftrightarrow \infty}=0
$$

where $\Delta H_{1 \leftrightarrow \infty}$ and $\Delta S_{1 \leftrightarrow \infty}$ are the changes of condensation enthalpy and of entropy, respectively, between the monomeric and bulk phases according to reactions $1-5$. At $T_{1 \leftrightarrow \infty}$, the interconversion (eq 7) of coexisting monomers to and from bulk,

$$
n \mathrm{M}_{1} \rightleftarrows \mathrm{M}_{n} \quad(n \rightarrow \infty)
$$

depends on the external energy exchange of the system and on the change of heat capacity between phases.

Although the mechanism $1-5$ and the overall equilibrium reaction 7 seem to be symmetrical with respect to thermodynamics, the transition is easier from order to chaos (evaporation from $M_{\infty}$ to $M_{1}$ ) than in the other way (condensation of $M_{1}$ into

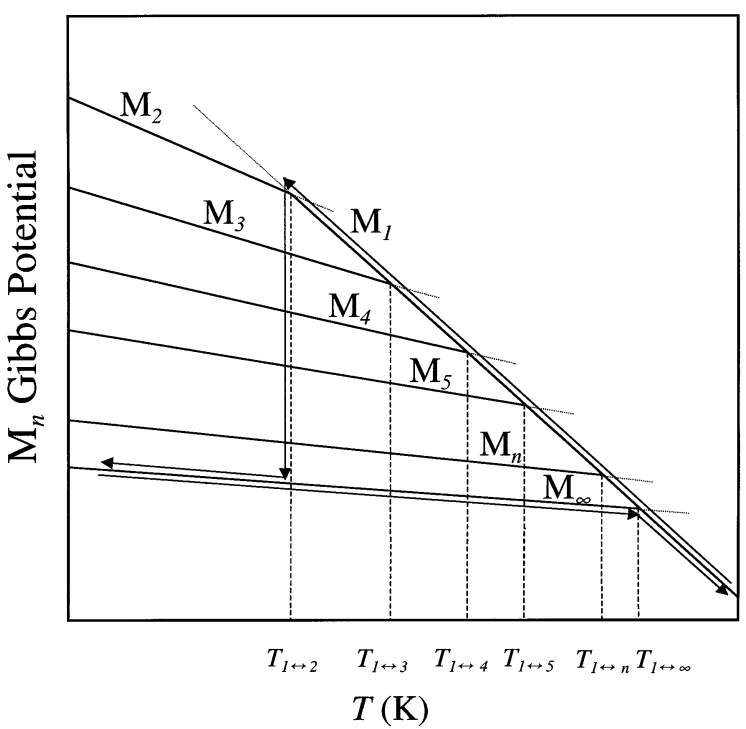

Figure 1. Temperature dependence of the free energy for the monomers, oligomers of increasing nuclearity, and condensed phase. At a given temperature, the free energy of $\mathbf{M}_{n}$ decreases for increasing $n$ below the bulk transition temperature. The temperature $T_{1 \leftrightarrow n}$, at which free energies for monomers and $\mathrm{M}_{n}$ are equal, increases with $n$. Because of the nuclearity dependence of the potential and of the negligible probability that the condensation of monomers starts but by the dimerization, the monomers are stable as far as $T_{1 \leftrightarrow 2}<T$. A thermal hysteresis appears between the phase-transition temperatures $T_{1 \rightarrow \infty}$ and $T_{\infty \rightarrow 1}$ in the course of decreasing and increasing temperature, respectively (see text).

$\mathrm{M}_{\infty}$ ), and the kinetics can be highly different. Actually, it is well-known that in a large temperature domain of metastability below $T_{1 \leftrightarrow \infty}$, the condensation could not occur at a detectable rate.

In the present phenomenological model, we take into account that (i) the process of monomer condensation is a multistep mechanism as in eqs $1-5$, which starts, in the absence of any preexisting oligomer, from the dimerization (eq 1) and (ii) the mean binding energy between the atoms $\mathbf{M}_{1}$ in a cluster $\mathbf{M}_{n}$ or the enthalpy of the $\mathbf{M}_{n}$ formation relative to $n$ free monomers (reaction 7) increases markedly with the nuclearity $n,,^{32,37-41}$ the variation depending on the nature of the considered system $\mathrm{M}$. That means that the very first step of the monomer condensation kinetics, which is the dimerization, also undergoes the highest activation barrier. At a given temperature, the enthalpy and the entropy of the cluster $\mathrm{M}_{n}$ decrease with $n$. In other words, if the intermediate cluster $\mathrm{M}_{n}$ were stable, an equation analogous to eq 6 could be written with nuclearity-dependent terms:

$$
\Delta G_{1 \leftrightarrow n}=\Delta H_{1 \leftrightarrow n}-T_{1 \leftrightarrow n} \Delta S_{1 \leftrightarrow n}=0
$$

We assume that the entropy and enthalpy of $\mathbf{M}_{n}$ are constant with $T$ and at a given $T$ both decrease with $n$. Thus for each $n$ value, the Gibbs potential of $\mathrm{M}_{n}$ decreases linearly with increasing $T$ and decreases at a given $T$ when $n$ increases. In Figure 1 are presented the variations with $T$ of the Gibbs potential of clusters of various nuclearity $n$. The free energy change $\Delta G_{1 \leftrightarrow n}$ in eq 8 is, for example, given by the potential difference between the lines for $\mathrm{M}_{1}$ and $\mathrm{M}_{n}$. As the value of $n$ becomes lower, the transition temperature $T_{1 \leftrightarrow n}$ between the cluster $\mathrm{M}_{n}$ and the monomer $\mathrm{M}_{1}$, at which their potentials are equal (crossing point between $\mathrm{M}_{1}$ and $\mathrm{M}_{n}$ potential lines), should become lower. The transition temperature of the macroscopic state $T_{1 \leftrightarrow \infty}$ is the upper limit of $T_{1 \leftrightarrow n}$. The stabilization of nanoclusters, of metal, for example, was experimentally obtained 
under some confined conditions, of deposition ${ }^{42,43}$ or in nanometric pores ${ }^{44}$ and indeed the melting point was found to decrease with a decrease in the cluster nuclearity and to be much lower than that of the bulk metal.

Because the condensation mechanism 1-5 should start kinetically with the dimer formation (reaction), the transition temperature $T_{1 \leftrightarrow 2}$ for $n=2$, which is the lowest value of $T_{1 \leftrightarrow n}$, is also the determining temperature required to observe some dimer formation. Thus, the nuclearity dependence of the cluster thermodynamical properties introduces necessarily an asymmetry between the kinetics of condensation and evaporation and a thermal hysteresis of the transition points $T_{\infty \rightarrow 1}$ and $T_{1 \rightarrow \infty}$.

In the domain $T_{1 \leftrightarrow \infty} \leq T$, the thermodynamical conditions are fulfilled for the dissociation into monomers of the bulk state and $a$ fortiori of all smaller nuclearity clusters. $\mathbf{M}_{\infty}$ is unstable above $T_{1 \leftrightarrow \infty}$. In Figure 1, the variation of the Gibbs potential of $\mathbf{M}_{n}$ is presented in this temperature range as a dotted line. For a system initially consisting of clusters $\mathbf{M}_{n}$, the evaporation indeed predominates over condensation because $T$ is already beyond $T_{1 \leftrightarrow n}$, which corresponds to the transition temperature of $\mathbf{M}_{n}$. The evaporation transition starts readily without any activation barrier.

In the temperature range $T<T_{1 \leftrightarrow 2}$, the formation of a dimer $\mathrm{M}_{2}$ among a monomer $\mathrm{M}_{1}$ population predominates over dimer dissociation and the conditions are even more favorable for the successive following steps of absorption of monomers by the clusters. The transition of the condensation of monomers occurs without any activation barrier. In this temperature range, the variation of the Gibbs potential of $\mathrm{M}_{1}$ is presented in Figure 1 as a dotted line. Under these conditions of a coalescence model with negligible evaporation and with a condensation rate constant independent of $n$, it was shown by Smoluchowski ${ }^{1}$ that the whole set of kinetics giving the time dependence of the concentration of any cluster $\mathbf{M}_{n}$ is invariant if the time is normalized as $t / \tau$ (with $\tau=1 /\left(k_{\mathrm{cond}} C_{0}\right)$ ), whatever are the initial concentration $C_{0}$ of $\mathrm{M}_{1}$ and the condensation rate constant $k_{\text {cond. }}{ }^{20,35,36}$

In the domain $T_{1 \leftrightarrow 2}<T<T_{1 \leftrightarrow \infty}$, for a system initially consisting of monomers only (prepared, for example, at temperature higher than $T_{1 \leftrightarrow \infty}$ and then cooled), the evaporation of $\mathrm{M}_{2}$ into $\mathrm{M}_{1}$ predominates over the dimer formation, and the probability is weak that a newly formed dimer is long-lived enough to let it produce $\mathrm{M}_{3}$ by absorbing another monomer and thus to start the growth. Therefore, in this temperature range, although $\mathbf{M}_{\infty}$ and all oligomers larger than the dimer are thermodynamically more stable than $\mathrm{M}_{1}$ because of their lower potentials, the monomers are kinetically stable, that is, are metastable. The system is supersaturated. Thus $T_{1 \leftrightarrow 2}$ constitutes the lower limit of the domain of metastability of monomers. The critical nucleus size for homogeneous nucleation is indeed $n=2$.

Thermal Hysteresis. In Figure 1, when the temperature is increased from the lowest values, the potential of the most stable state $\mathrm{M}_{\infty}$ decreases and continues to decrease until $T_{1 \leftrightarrow \infty}$, when it is replaced by that of $\mathrm{M}_{1}$. This may be achieved by a cascade of first-order fragmentation reactions, which are all thermodynamically and kinetically favored at $T_{1 \leftrightarrow \infty} \leq T$. In contrast, when the temperature is decreased from the high values, the potential of the monomers $\mathrm{M}_{1}$ should increase up to the potential value at $T_{1 \leftrightarrow 2}$ at which they become unstable with respect to dimerization. As soon as the dimerization is thermodynamically allowed, it occurs and initiates the following condensation cascade to larger (and transient) clusters, which exhibit an increasing stability in this temperature range (mechanism 1-5,

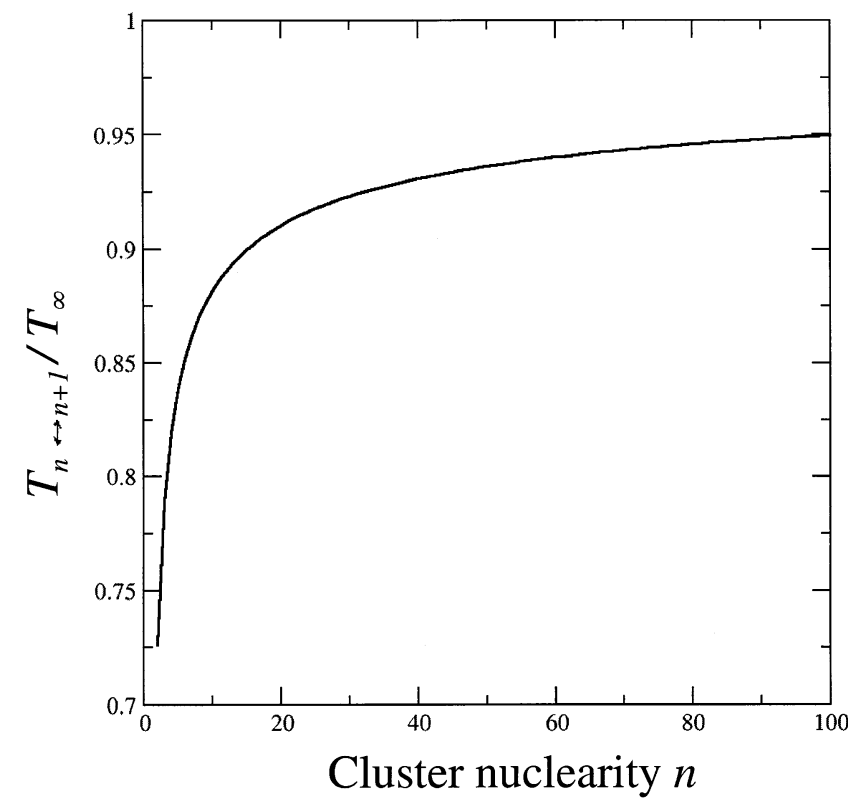

Figure 2. Nuclearity dependence of the transition temperature between $\mathbf{M}_{n}$ and $\mathbf{M}_{n+1}$. The temperature is normalized to the transition temperature of the bulk.

forward). Thus the potential decreases suddenly in the replacement of $\mathrm{M}_{1}$ by the condensed phase $\mathrm{M}_{\infty}$. At lower temperature, the potential increases less markedly, as does the $\mathrm{M}_{\infty}$ potential.

The asymmetry of the potential change in the course of increasing and decreasing temperature is due to both (i) the nuclearity dependence of the oligomer Gibbs potential (decreasing with increasing $n$ at $T<T_{1 \leftrightarrow \infty}$ ) and (ii) the negligible probability that the condensation would start without a dimerization, so introducing a kinetical barrier, a supersaturation, and a thermal hysteresis in the phase transition $\left(T_{1 \rightarrow \infty} \ll T_{\infty \rightarrow 1}\right)$.

\section{Nuclearity Dependence of the Transition Temperature between Consecutive Oligomers}

Recently, some calculations have been achieved by different methods on the thermodynamics of variable nuclearity oligomer series. Neutral silver ${ }^{32,37-39}$ or water ${ }^{40,41,45,46}$ clusters are among the most studied. The different calculation approaches, ab initio, ${ }^{32}$ density functional theory (DFT), ${ }^{37,38}$ or molecular dynamics (MD), ${ }^{39}$ are converging on the general trend with $n$ of the nuclearity dependence of the clusters $\mathrm{M}_{n}$ average binding energy per atom, $E_{\mathrm{b}}(n)$, as that used by Erkoç and Yilmaz. ${ }^{39}$

As a first approximation, we derive from this $E_{\mathrm{b}}(n)$ function the enthalpy change from $\mathbf{M}_{n}$ to $\mathbf{M}_{n+1}$ :

$$
\Delta H_{n \leftrightarrow n+1}=\Phi+\alpha\left[(n+1)^{2 / 3}-n^{2 / 3}\right]
$$

where $\Phi$ is the bulk cohesive energy of the condensed phase and $\alpha=2^{1 / 3}\left(\Delta H_{1 \leftrightarrow 2} / 2-\Phi\right), \Delta H_{1 \leftrightarrow 2}$ being the dimer binding energy.

We assume a similar variation for $\Delta S_{n \leftrightarrow n+1}$ and calculate the transition temperature $T_{n \leftrightarrow n+1}=\Delta H_{n \leftrightarrow n+1} / \Delta S_{n \leftrightarrow n+1}$ between $\mathbf{M}_{n}$ and $\mathbf{M}_{n+1}$. In Figure 2 is shown the nuclearity dependence of the ratio of $T_{n \leftrightarrow n+1}$ to its limit $T_{\infty}$. The overall variation of the ratio is about $25 \%$ between $n=2$ and the bulk. The steep variation of $T_{n \leftrightarrow n+1}$ with $n$ for low $n$ values indicates clearly the influence of $n$ on the successive transitions by which the initial dimers will grow up to the bulk. Conversely speaking, if a temperature $T_{n \leftrightarrow n+1}$ is imposed to the clusters of size $n+1$, smaller clusters are also unstable with respect to evaporation yielding eventually to the monomer phase. This confirms that 
the value of $T_{1 \leftrightarrow 2}$ is the lower limit of the metastability domain for monomers under supercritical conditions of temperature.

\section{Conclusion}

The phenomenological model of homogeneous nucleation, or of the build-up of a cluster from a monomeric phase, which is presented here, takes into account the nuclearity-dependent properties of clusters, particularly of the dimers and the smallest oligomers. With this respect, the nuclearity dependence of the binding energy obtained by recent theoretical calculations is crucial.

The thermal hysteresis of the phase transition, concerning, for example, the temperature of condensation from monomers to the bulk compared to the evaporation temperature from the bulk to monomers, and the existence of a temperature domain in which monomers are metastable result from the increasing binding energy with $n$ of the supplementary monomer, combined with the fact that the dimer formation is the rate-determining step of the process. The difference in binding energy between $\mathrm{M}_{2}$ and $\mathrm{M}_{n}$ creates an activation barrier for the condensation of monomers into $\mathbf{M}_{n}$. This variable binding energy also accounts for the nuclearity dependence of the evaporation temperature of finite-size clusters.

Acknowledgment. The authors gratefully acknowledge Professor Jacques Friedel for stimulating discussion. This work is dedicated to Prof. Arnim Henglein with whom J.B. shared for decades the same steady interest in cluster science and with whom the exchanges were the most fruitful.

\section{References and Notes}

(1) Smoluchowski, M. Phys. Z. 1916, 17, 385.

(2) Volmer, M.; Weber, A. Z. Phys. Chem. 1926, 119, 227.

(3) Becker, R.; Dohring W. Ann. Phys. 1935, 24, 719.

(4) Zeldovich, J. B. J. Exp. Theor. Phys. 1942, 0, 525.

(5) Turnbull, D.; Fisher, J. C. J. Chem. Phys. 1949, 17, 71. 1969.

(6) Zettlemoyer, A. C., Ed. Nucleation; Marcel Dekker: New York,

(7) Castleman, A. W. Space Sci. Rev. 1974, 15, 547.

(8) Chernov, A. A. Modern crystallography, III Crystal Growth; Springer-Verlag: Berlin, 1984.

(9) Oxtoby, D. W. In Fundamentals of inhomogeneous fluids; Henderson, D., Ed.; Marcel Dekker: New York, 1992; p 407.

(10) Stoyanov, S.; Kashchiev, D. Thin film nucleation and growth theories: a confrontation with experiments; Current Topics in Materials Science, Vol. 7; North-Holland: Amsterdam, 1981; p 69.

(11) Mandell, M. J.; McTague, J. P.; Rahman, A. J. Chem. Phys. 1976, 64, 3699.

(12) Hsu, C. S.; Rahman, A. J. Chem. Phys. 1979, 70, 5234; 1979, 71, 4974.
(13) Fuchs, A. H.; Pawley, G. S. J. Phys. 1988, 49, 41.

(14) Kunz, R.; Berry, R. S. Phys. Rev. 1994, 49, 1895.

(15) Baxendale, J. H.; Fielden, E. M.; Keene, J. P.; Ebert, M. In Pulse Radiolysis; Keene, J. P., Swallow, A., Baxendale, J. H., Eds.; Academic Press: London, 1965; p 207.

(16) Von Pukies, J.; Roebke W.; Henglein, A. Ber. Bunsen-Ges. Phys. Chem. 1968, 72, 842 .

(17) Tausch-Treml, R.; Henglein, A.; Lilie, J. Ber. Bunsen-Ges. Phys. Chem. 1978, 82, 1335 .

(18) Belloni, J.; Delcourt, M. O.; Marignier, J. L.; Amblard, J. In Radiation Chemistry; Hedwig, P., Nyikos, L., Schiller, R., Eds.; Akad. Kiado: Budapest, Hungary, 1987; p 89.

(19) Swayambunathan, V.; Hayes, D.; Schmidt, K. H.; Liano, Y. X. Meisel, D. J. Am. Chem. Soc. 1990, 112, 3831.

(20) Mostafavi, M.; Liu, Yun-Ping; Pernot, P.; Belloni, J. Radiat. Phys. Chem. 2000, 59, 49.

(21) Schmidt, K. H.; Patel, R.; Meisel, D. J. Am. Chem. Soc. 1988, 110 , 4882 .

(22) Zhang, H. G.; Mostafavi, M. J. Phys. Chem. B 1997, 101, 8443.

(23) Delcourt, M. O.; Belloni, J. Radiochem. Radioanal. Lett. 1973, 13 , 329.

(24) Henglein, A. Ber. Bunsen-Ges. Phys. Chem. 1977, 81, 556.

(25) Henglein, A. Chem. Rev. 1989, 89, 1861; Ber. Bunsen-Ges. Phys. Chem. 1995, 99, 903

(26) Belloni, J.; Mostafavi, M. In Studies in physical and theoretical Chemistry. Radiation Chemistry: Present status and future trends; Jonah

C. D., Rao, M., Eds.; Elsevier: Amsterdam, 2001; Vol. 87, p 411.

(27) Kubo, R. J. Phys. Soc. Jpn. 1962, 17, 975.

(28) Halperin, W. H. Rev. Mod. Phys. 1986, 58, 533.

(29) Morse, M. D. Chem. Rev. 1986, 86, 1046

(30) Schumacher, E. Chimia 1988, 42, 357.

(31) Haberland, H., Ed. Clusters of atoms and Molecules; Springer: Berlin, 1994.

(32) Bonacic-Koutecky, V.; Cespiva, L.; Fantucci P.; Koutecky, J. Z Phys. D 1993, 26, 287.

(33) Moisar, E. In Contribution of clusters physics to materials science and technology; Davenas, J., Rabette, P. M., Eds.; NATO ASI Series E, Applied Sciences no. 104; Nijohff: Dordrecht, Netherlands, 1986; p 311.

(34) Malinowski, J. In Growth and properties of metal clusters; Bourdon, J., Ed.; Elsevier: Amsterdam, 1980; p 303.

(35) Mostafavi, M.; Marignier, J. L.; Amblard, J.; Belloni, J. Radiat Phys. Chem. 1989, 34, 605; J. Imaging Sci. 1991, 35, 68.

(36) Khatouri, J.; Mostafavi, M.; Amblard, J.; Ridard, J.; Belloni, J. Z. Phys. D Atoms, Mol. Clusters 1993, 26, 82. Note that odd-even effects observed in these simulations result from an overestimation by 2 of quadratic terms.

(37) Santamaria, R.; Kaplan, I. G.; Novaro, O. Chem. Phys. 1994, 218, 395.

(38) Poteau, R.; Heully, J. L.; Spiegelman, F. Z. Phys. D 1997, 40, 479.

(39) Ercoç, S.; Yilmaz, T. Physica E 1999, 5, 1

(40) Quintana, I. M.; Ortiz, W.; Lopez, G. E. Chem. Phys. Lett. 1998, 287, 429 .

(41) Erkoç, S; Güneyler, E. Physica E 2000, 8, 40

(42) Buffat, P. A. Thin Solid Films 1976, 32, 283

(43) Borel, J. P. Surf. Sci. 1981, 106, 1.

(44) Dick, K.; Dhanasekaran, T.; Zhang, Z. Y.; Meisel, D. J. Am. Chem. Soc. 2002, 124, 2312.

(45) Xantheas, S. S. Chem. Phys. 2000, 258, 225.

(46) Ocasio, M.; Lopez, G. E. Chem. Phys. Lett. 2002, 356, 168. 